\title{
Tensor based structure estimation in multi-channel images
}

\section{Schou, Jesper; Dierking, Wolfgang; Skriver, Henning}

\section{Published in:}

Proceedings on Geoscience and Remote Sensing Symposium

Link to article, DOI:

10.1109/IGARSS.2000.861664

Publication date:

2000

Document Version

Publisher's PDF, also known as Version of record

Link back to DTU Orbit

Citation (APA):

Schou, J., Dierking, W., \& Skriver, H. (2000). Tensor based structure estimation in multi-channel images. In Proceedings on Geoscience and Remote Sensing Symposium (Vol. 2, pp. 663-665). IEEE.

https://doi.org/10.1109/IGARSS.2000.861664

\section{General rights}

Copyright and moral rights for the publications made accessible in the public portal are retained by the authors and/or other copyright owners and it is a condition of accessing publications that users recognise and abide by the legal requirements associated with these rights.

- Users may download and print one copy of any publication from the public portal for the purpose of private study or research.

- You may not further distribute the material or use it for any profit-making activity or commercial gain

- You may freely distribute the URL identifying the publication in the public portal

If you believe that this document breaches copyright please contact us providing details, and we will remove access to the work immediately and investigate your claim. 


\title{
Tensor Based Structure Estimation in Multi-channel images
}

\author{
J. Schou, W. Dierking, and H. Skriver \\ Department of Electromagnetic Systems, Technical University of Denmark, DK-2800 Lyngby, Denmark \\ Facsimile: +45 45931634 \\ e-mail: js@emi.dtu.dk,wd@emi.dtu.dk, hs@emi.dtu.dk
}

\begin{abstract}
In this work a two-step algorithm is proposed for detecting structures in multi-channel remote sensing images. The first part is a normal low level structure detection performed on the individual images, which provides information of the strength as well as the orientation of the structure. In the second part tensors are used for representing the structure information. This approach has the advantage, that tensors can be averaged either spatially or by applying several images, and the resulting tensor provides information of the average strength as well as orientation of the structure. The performance of the algorithm is examined using multi-frequency, polarimetric SAR images from the Danish airborne EMISAR.
\end{abstract}

\section{INTRODUCTION}

Detection of structures in remote sensing images is relevant for many applications, e.g. detection of man-made structures such as road networks [1], or detection of boundaries between land-use classes as an initial step for segmentation [2]. Several structure detectors have been developed taking the statistical properties of the images into account, for optical as well as synthetic aperture radar (SAR) data, but most of these work on single-channel images. When examining multi-channel data, e.g. multi-temporal data or data acquired at different frequencies or polarizations, the normal approach is to examine the images individually, and subsequently combine the results using one of several fusion operators [3][1][2].

In this work we are concerned with detection of line- and edge-structures, ie. structures that are described by their strength as well as orientation in the images. The strength of a structure is defined according to the statistic of the image; for optical images it is the difference of averages in a local neighborhood, while SAR images requires ratios of averages to obtain constant false alarm rates [4][5].

The normal fusion operator only utilizes the strength of the structure, while the tensor based approach in this work includes the structure orientation as well. We argue, that the orientation information is an important parameter, as a structure having the same orientation in a set of images seems more likely than a structure detected at different orientations in the same set of images. The tensor representation has previously been applied in computer vision, and described by, e.g., Granlund and Knutsson [6] and Knutsson et al. [7].

\section{LOW LEVEL STRUCTURE DETECTION}

The tensor based structure estimation algorithm applies a low level structure detection to the images in the first part of the algorithm. The only constraints on the choice of detector are, that it must provide information of the strength as well as orientation of the local structure, and that it should be adapted to the statistic of the images applied.

In this work we examine linear structures in polarimetric SAR data. The data can be described by the polarimetric covariance matrix $\mathbf{C}$

$$
\mathbf{C}=\left[\begin{array}{ccc}
\sigma_{h h} & \sqrt{\sigma_{h h} \sigma_{h v}} \rho_{h h, h v} & \sqrt{\sigma_{h h} \sigma_{v v}} \rho_{h h, v v} \\
\sqrt{\sigma_{h h} \sigma_{h v}} \rho_{h h, h v}^{*} & \sigma_{h v} & \sqrt{\sigma_{h v} \sigma_{v v} \rho_{h v, v v}} \\
\sqrt{\sigma_{h h} \sigma_{v v}} \rho_{h h, v v}^{*} & \sqrt{\sigma_{h v} \sigma_{v v}} \rho_{h v, v v}^{*} & \sigma_{v v}
\end{array}\right]
$$

with $\sigma_{j}$ being the backscattering coefficient for polarization $j$, $\rho_{j, k}$ the complex correlation coefficient of polarizations $j$ and $k$, and $*$ denoting complex conjugation. A natural choice of structure detector would hence be a detector capable of examining the full covariance matrix, but such a detector has not yet been developed. Instead we apply the ratio line detector suggested by Lopes et al. [8] working on the intensity images (the backscattering coefficients). It applies a set of filters working in a local $M \times M$ window, and if the ratio of the intensity of the central strip to the intensity of the neighboring areas is sufficiently large or small, a structure is detected. The strength is given by the intensity ratio, and the orientation by the orientation of the filter resulting in the highest ratio.

\section{ORIENTATION TENSOR}

The low level structure detection provides information of the orientation and strength of a structure at each pixel. In the following $\hat{\mathbf{x}}_{i}=\left(\begin{array}{c}\cos \varphi \\ \sin \varphi\end{array}\right)$ and $A_{i}$ denotes the orientation and strength at pixel $i$, respectively, with $\varphi$ belonging to the interval $\left[0, \pi\left[\right.\right.$, and $A_{i}$ to the interval $[0,1]$ with $A_{i}=1$ being full certainty of a structure present. The orientation tensor at pixel $i$ is defined as [6]

$$
\mathbf{T}_{i}=A_{i} \hat{\mathbf{x}}_{i} \hat{\mathbf{x}}_{i}^{t}
$$

with $t$ denoting transpose, and for a $2-\mathrm{D}$ image $\mathbf{T}_{\boldsymbol{i}}$ is a $2 \times 2$ tensor. The norm of the tensor equals $A_{i}$, and the norm is independent of the orientation represented by $\mathbf{T}_{i}$. 
The tensor representation is useful, because averaging of tensors is a meaningful operation, as mentioned by Knutsson et al. [7]. Averaging of a variable is generally performed to obtain an estimate having higher precision or certainty than the individual variable, and in the context of this work, it is done using one of the following two methods: 1) Using multiple images and averaging the tensors located at pixel $i$ in all the images; 2) applying a local averaging filter to a single tensor image. The first method is useful for data fusion, using e.g. multi-temporal data or data acquired at different frequencies or polarizations. The second method utilizes the spatial information of the structures in the images, and this latter method can be combined with the data fusion approach, resulting in a spatially averaged tensor from multiple images.

The averaging operation results in an estimate, $\overline{\mathbf{T}}_{i}$, which is based on a number of tensors generally having different orientations. For this reason (2) can not be used for describing $\overline{\mathbf{T}}_{i}$, but it is still possible to find the best approximation to $\overline{\mathbf{T}}_{i}$ which can be expressed using (2), as argued by Granlund and Knutsson [6]. This tensor, $\mathbf{T}_{a i}$, is found by minimizing the norm $\left\|\mathbf{T}_{a i}-\overline{\mathbf{T}}_{i}\right\|$ yielding [6]

$$
\mathbf{T}_{a i}=\lambda_{1 i} \hat{\mathbf{e}}_{1 i} \hat{\mathbf{e}}_{1 i}^{t}
$$

with $\lambda_{1 i}$ being the largest eigenvalue of the tensor $\overline{\mathbf{T}}_{i}$ and $\hat{\mathrm{e}}_{1 i}$ the corresponding eigenvector. In other words, the largest eigenvalue of the averaged orientation tensor provides the average structure strength at a given pixel, and the corresponding eigenvector provides the average orientation.

For a 2-D image two eigenvalues exist, and we apply the variable $C_{a i}$ to describe the quality of the approximation

$$
C_{a i}=\frac{\lambda_{1 i}}{\lambda_{2 i}}
$$

$C_{a i}$ belongs to the interval $\left[1, \infty\left[\right.\right.$ with $C_{a i} \rightarrow \infty$ corresponding to the perfect approximation.

Fig. 1 shows a geometric description of the average orientation tensor for three different cases. In each case the resulting tensor is the average of two tensors, $\mathbf{T}_{1}$ and $\mathbf{T}_{2}$, described by (2). In the first case, shown in Fig. 1 a), the average tensor is comprised of tensors having the same orientation, thus $\lambda_{2 i}=0$ and $C_{a i}$ becomes infinite corresponding to the perfect approximation. The norm of the average tensor equals the norm of the input tensors. When the two tensors have orthogonal orientations but equal norms, as in Fig. $1 \mathrm{~b}$ ), the two eigenvalues are equal making $C_{a i}=1$, and the tensor provides no information of the orientation of the structure. The norm of the average tensor becomes half that of the input tensors, illustrating the strength of the tensor approach compared to normal fusion operators which normally discard the information of the orientations; in this case discarding the orientation information results in an output value being equal to the input values (for the mean operator), but obviously the presence of a structure is less certain, when the input variables suggest orthogonal orientations. a)

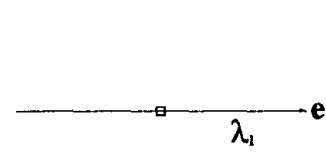

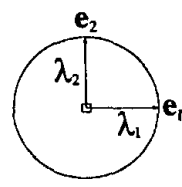

b)

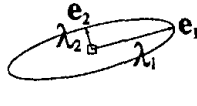

c)
Fig. 1: Geometric representation of average orientation tensor $\overline{\mathbf{T}}$. $\lambda_{1}$ is the largest eigenvalue and $\hat{\mathbf{e}}_{1}=\left(\begin{array}{c}\cos \bar{\varphi} \\ \sin \bar{\varphi}\end{array}\right)$ is the corresponding eigenvector having orientation $\bar{\varphi}$

(a) $A_{1}=A_{2}=A, \varphi_{1}=\varphi_{2}=0 \Rightarrow \lambda_{1}=A, \lambda_{2}=0, \bar{\varphi}=0$

(b) $A_{1}=A_{2}=A, \varphi_{1}=0, \varphi_{2}=\frac{\pi}{2} \Rightarrow \lambda_{1}=\lambda_{2}=\frac{A}{2}, \bar{\varphi}$ not defined

(c) $A_{1}=2 A_{2}=A, \varphi_{1}=0, \varphi_{2}=0.4 \pi \Rightarrow \lambda_{1}=0.59 A$,

$\lambda_{2}=0.16 A, \bar{\varphi}=\frac{\pi}{12}$

Fig. $1 \mathrm{c}$ ) illustrates the most common case, where the input tensors are neither parallel nor orthogonal and the norms are different, and the average tensor is a compromise based on the strengths and orientations of the input tensors.

\section{EXPERIMENTAL RESULTS}

Fig. 2 shows a 13 -look $h v$ polarized intensity image from the Danish EMISAR [9], having a $5 \times 5 \mathrm{~m}$ ground pixel spacing. We examine the algorithm on the small area in the box, including a road and surrounding fields. In Fig. 3(a) is shown the result when applying the low level line detector on the $h v$ polarized image. The dimensions and orientations of the lines corresponds to the line strengths and line filters resulting in highest strength, respectively, and only the pixels resulting in line strengths above a threshold are included. The effects of the

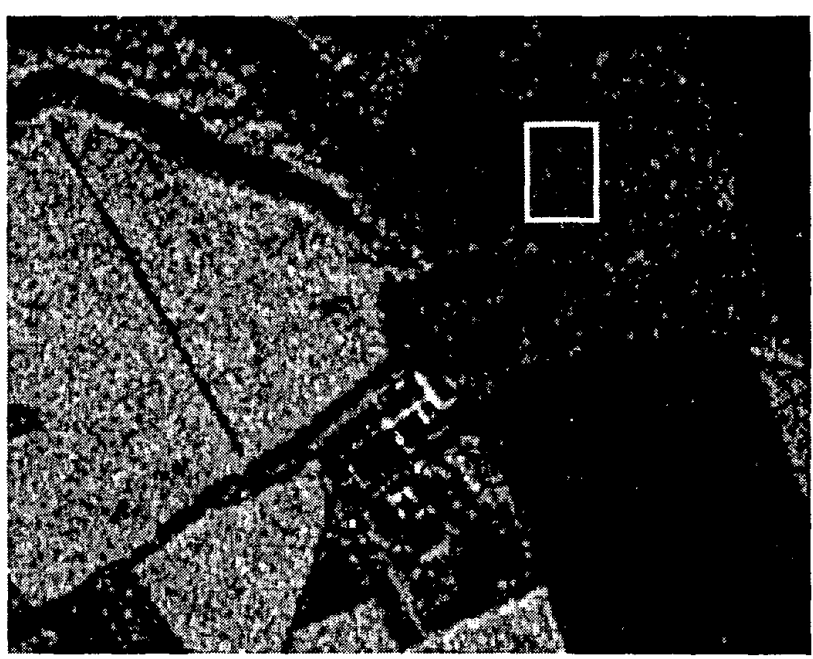

Fig. 2: C-band EMISAR image, $\sigma_{h v}$ including test area 


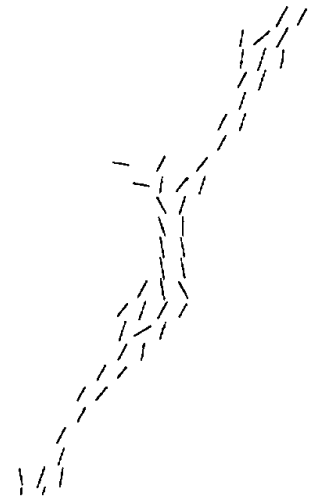

(a)

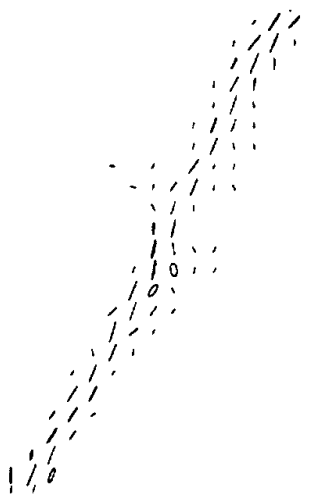

(c)

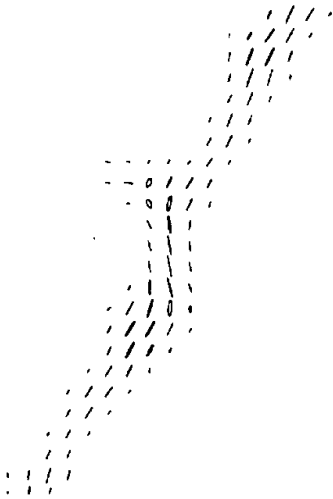

(b)

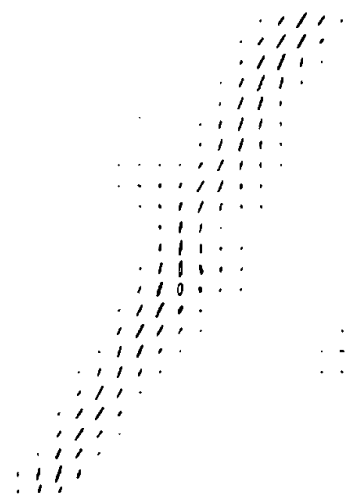

(d)
Fig. 3: Result of tensor based line detection using EMISAR data. a) result of low level line detection using $h v$ polarization for test area, b) average line result using $2 \times 2$ spatially averaging of $h v$ polarization, c) average line result using polarizations $h h, h v$ and $v v$, d) average line result using $2 \times 2$ spatially averaging of $h h, h v$ and $v v$.

tensor based averaging are seen in Fig. 3(b) to (d), using spatial averaging and/or averaging of multiple polarizations and the geometric representation from Fig. 1. We observe, as expected, that the more information used in the filtering the more smooth the resulting line estimates become. It is also seen, that the filtering results in several line estimates having a very small line strength, and a second line strength thresholding might be applied.

The tensor based structure estimation can be used by all applications using information of structures, but especially applications using information of the orientation of the structures benefit from the approach. Currently, a road detection scheme based on an active contour model [10] is being developed, using the average structure information shown in Fig. 3.

\section{CONCLUSION}

An algorithm for estimating structures in remote sensing images is presented, using a low level structure detector in the first step, and a tensor based representation of the structure in the second step. Compared to the normal fusion operators, the approach makes it possible to perform averaging, spatially as well as on different data sets, using the strength as well as orientation of the detected structures. The resulting structure estimate provides information of the average structure strength as well as orientation, thus making it very suited for applications using the structure orientations.

\section{REFERENCES}

[1] F. Tupin, H. Maitre, J.-F. Mangin, J.-M. Nicolas, and E. Pechersky "Detection of Linear Features in SAR Images: Application to Road Network Extraction", IEEE Transactions on Geoscience and Remote Sensing, Vol. 36, No. 2, March, 1998 pp. 434-453

[2] C. Oliver and S. Quegan "Understanding Synthetic Aperture Radar Images", Artech House, 1998

[3] I. Bloch "Information combination operators for data fusion: A comparative review with classification" , IEEE Transactions on Systems, Man \& Cybernetics, Part A, Vol. 26, Issue 1, 1996 pp. 52-67

[4] S.N. Madsen "Speckle Theory: Modelling, analysis, and applications related to Synthetic Aperture Radar data" Ph.D. thesis, No. LD 62, Department of Electromagnetic Systems, Technical University of Denmark, November, 1986

[5] R. Touzi, A. Lopes, and P. Bousquet "A Statistical and Geometrical Edge Detector for SAR Images", IEEE Transactions on Geoscience and Remote Sensing, Vol. 26, No. 6, November, 1988 pp. 764-773

[6] G.H. Granlund and H. Knutsson "Signal Processing for Computer Vision", Kluwer Academic Publishers, 1995

[7] H. Knutsson, L. Haglund, H. Bårman and G.H. Granlund "A Framework for Anisotropic Adaptive Filtering and Analysis of Image Sequences and Volumes", ICASSP-92, Vol.3, 1992 pp. 469-472

[8] A. Lopes, E. Nezry, R. Touzi, and H. Laur "Structure detection and statistical adaptive speckle filtering in SAR images", Int. J. Remote Sensing, Vol. 14, No. 9, 1993 pp. 1735-1758

[9] E.L. Christensen, N. Skou, J. Dall, K.W. Woelders, J.H. Jørgensen, J. Granholm and S.N. Madsen "EMISAR: An Absolutely Calibrated Polarimetric L- and C-band SAR" IEEE Transactions on Geoscience and Remote Sensing, Vol. 36, No. 6, November, 1998 pp. 1852-1865

[10] M.S. Horritt "A statistical active contour model for SAR image segmentation", Image and Vision Computing, Vol. 17, Issue 3-4, 1999 pp. 213-224 